\title{
Tunable and Efficient Red to Near-Infrared Photoluminescence by Synergistic Exploitation of Core and Surface Silver Doping of CdSe Nanoplatelets
}

\author{
Ali Hossain Khan, ${ }^{\dagger, \ddagger \odot ~ V a l e r i o ~ P i n c h e t t i, ~}{ }^{\S}$ I Ivo Tanghe, ${ }^{\dagger, \|, \perp}$ Zhiya Dang, Beatriz Martín-García, \\ Zeger Hens, ${ }^{\dagger, \perp}$ Dries Van Thourhout, ${ }^{\|, \perp}$ Pieter Geiregat, ${ }^{\dagger, \perp}{ }^{\circ}$ Sergio Brovelli, ${ }^{\S}{ }^{\circledR}$ \\ and Iwan Moreels $*, \dagger, \ddagger$ (0) \\ ${ }^{\dagger}$ Department of Chemistry, Ghent University, Krijgslaan 281-S3, 9000 Ghent, Belgium \\ ${ }^{\ddagger}$ Istituto Italiano di Tecnologia, via Morego 30, 16163 Genova, Italy \\ ${ }^{\S}$ Dipartimento di Scienza dei Materiali, Universitá degli Studi di Milano-Bicocca, Via Cozzi 55, 20125 Milano, Italy \\ "Photonics Research Group, INTEC Department, Ghent University-IMEC, Technologiepark-Zwijnaarde 15, 9052 Zwijnaarde, \\ Belgium \\ ${ }^{\perp}$ Center for Nano- and Biophotonics (NB-Photonics), Ghent University, Technologiepark-Zwijnaarde 15, 9052 Zwijnaarde, \\ Belgium
}

\section{Supporting Information}

ABSTRACT: We report on the synthesis of silver (Ag)doped CdSe nanoplatelets (NPLs) via postsynthesis cation exchange, using silver acetate as the Ag precursor. Highresolution transmission electron microscopy and X-ray diffraction confirmed that the NPLs maintain their morphology and crystal structure after doping when executing the exchange under reduced temperature in an ice bath. Spectroelectrochemistry and transient absorption spectroscopy revealed that $\mathrm{Ag}^{+}$acts as an acceptor dopant. $\mathrm{Ag}$ doping results in an emission that is tunable from 609 to $880 \mathrm{~nm}$, with a Stokes shift up to $1 \mathrm{eV}$ and a photoluminescence quantum efficiency exceeding 50\%. This is achieved by varying the Ag dopant concentration, which determines the hole energy level, and by controlling the electron energy level via quantum confinement in CdSe NPLs with varying core thickness or in CdSe/CdS core/shell NPLs. As highly fluorescent materials with a strongly suppressed emission reabsorption because of the large Stokes shift, Ag-doped colloidal two-dimensional NPLs offer new opportunities for the development of colloidal nanocrystal-based optoelectronic and photonic devices such as light-emitting diodes or luminescent solar concentrators.

\section{INTRODUCTION}

Colloidal nanocrystals (NCs) are solution-processed nanomaterials that have tunable optoelectronic properties because of the size-dependent quantum confinement effect. ${ }^{1,2}$ This enables efficient band-edge (BE) emission over a wide spectral range, from the ultraviolet to the near-infrared. ${ }^{1}$ Continuous progress in the field has yielded NCs with diverse shapes, from spherical quantum dots (QDs), to anisotropic, radially confined nanorods, tetrapods, and octapods, and two-dimensional (2D) nanoplatelets (NPLs). ${ }^{3}$ With shape control, one can precisely engineer the NC band structure and the associated optoelectronic properties such as the absorption cross section, the $\mathrm{BE}$ oscillator strength and exciton recombination time, the polarization of absorption and emission, and carrier relaxation dynamics in linear and nonlinear excitation regimes. ${ }^{1,4}$

Further band structure modifications occur when we include small amounts of dopants, capable of introducing atomic-like energy levels in the band gap of the host, which participate in
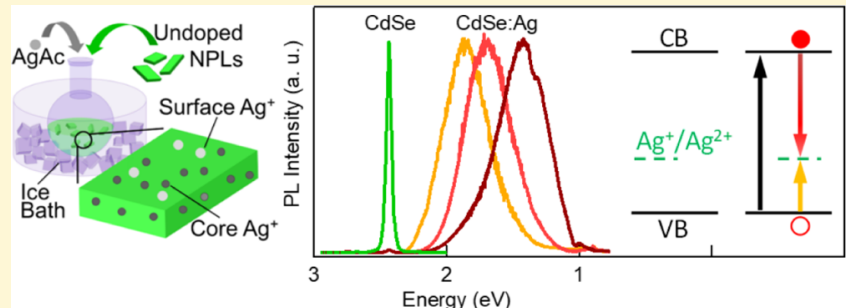

the NC photophysics either as acceptor/donor states for excited carriers or as color centers sensitized by the NC matrix. In this way, the Stokes shift between the host absorption edge and the dopant-mediated emission is increased significantly, yielding bright NCs with a strongly reduced self-absorption. ${ }^{5}$ Consequently, doped NCs have already been developed as potential candidates for application in luminescent solar concentrators (LSCs). ${ }^{5-7}$ By localizing one or both charge carriers, the exciton lifetime is extended, as the electron-hole wave function overlap decreases. The long lifetime of the dopant emission, exceeding the typical biological background fluorescence, makes doped NCs convenient biosensing or bioimaging markers, ${ }^{8}$ and in QD-sensitized solar cells it boosts the energy conversion efficiency. ${ }^{9}$ In addition, charge-carrier localization can improve the single-photon emission character-

Received: December 27, 2018

Revised: January 29, 2019

Published: January 30, 2019 
a)
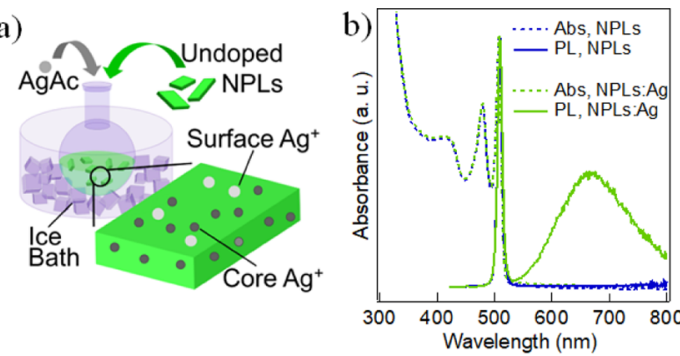

d)
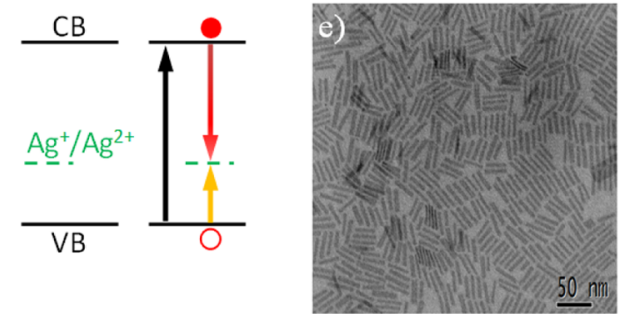
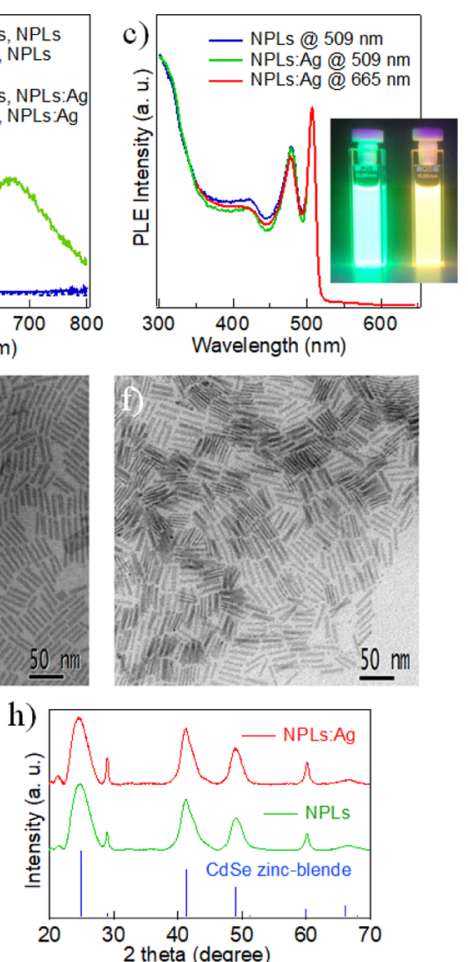

g)

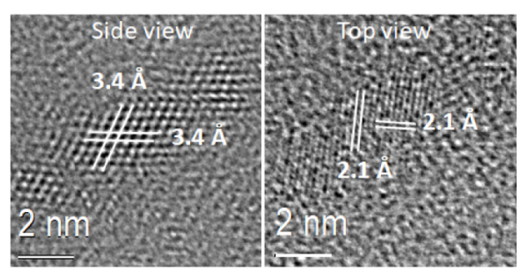

Figure 1. (a) Schematic representation of the cation exchange reaction, including the final CdSe:Ag NPLs. (b) Absorbance (dashed lines) and steady-state PL (solid lines) spectra of CdSe (blue) and CdSe:Ag NPLs (green), prepared by exposing the CdSe NPLs to silver acetate at a 0.2:1 $\mathrm{Ag} / \mathrm{Cd}$ molar ratio. (c) PL excitation spectra of CdSe NPLs (blue) and CdSe:Ag NPLs measured at the BE (green) and dopant emission (red), respectively. The inset shows the images of the CdSe and CdSe:Ag NPL dispersions under UV illumination. (d) Schematic presentation of the relevant CdSe:Ag NPL energy levels. Black, yellow, and red arrows mark the photon absorption/electron-hole creation, hole relaxation into the Ag dopant, and electron-hole recombination process, respectively. (e-f) TEM images of the corresponding CdSe (e) and CdSe:Ag (f) NPLs. (g) HRTEM images, including a side view (left panel) and a top view (right panel) of the CdSe:Ag NPLs (prepared with an Ag/Cd ratio of 0.2:1). (h) XRD patterns of CdSe and CdSe:Ag NPLs. Vertical lines indicate bulk zinc-blende CdSe (ICDD card no. 98-018-6011).

istics of the extended 2D CdSe NPLs. ${ }^{10}$ The broad dopant emission spectra make doped NCs potential candidates for multicolor or white light-emitting diodes (LEDs), $)^{5,11,12}$ and, in the nonlinear emission regime, repulsive Coulomb interactions between multiple localized carriers in doped NCs yield a strongly blue-shifted biexciton emission, ${ }^{13}$ which may lead to the realization of novel gain materials.

Isovalent doping of II-VI chalcogenide NCs, and more recently perovskite $\mathrm{NCs}$, using manganese $(\mathrm{Mn})$ has produced one of the most widely studied doped NC emitters. ${ }^{5,7,14,15}$ Yet, Mn-doped NCs fluoresce over a limited spectral range (typically $580-620 \mathrm{~nm}$ ), as the emission originates from localized $d-d$ transitions. ${ }^{14}$ Some tuning can be achieved by modifying the local crystal field around the dopant or by replacing the surface ligands. ${ }^{16,17}$ Heterovalent or electronic NC doping with copper $(\mathrm{Cu})$, on the other hand, yields a broader range $\left(413-1100 \mathrm{~nm}\right.$ has been reported), ${ }^{5}$ as here only the holes are localized on the dopants, and the electron wave function remains delocalized over the entire NC and thus sensitive to quantum confinement. As $\mathrm{Cu}$ has two stable oxidation states, +1 and +2 , there are different literature reports on its oxidation state in $\mathrm{Cu}$-doped NCs. ${ }^{5}$ An alternative electronic dopant in II-VI NCs is presented by silver ( $\mathrm{Ag})$, which, in contrast with $\mathrm{Cu}$, remains in a +1 oxidation state as its second ionization potential is very high. ${ }^{18}$ Photoexcitation leads to deep hole trapping at $\mathrm{Ag}^{+}$that subsequently forms $\mathrm{Ag}^{2+}$, which acts as a radiative acceptor of a conduction band (CB) electron, resulting in a strong Stokes-shifted dopant emission. ${ }^{19}$ As both the tetrahedral and octahedral ionic radii of $\mathrm{Cd}^{2+}(0.78$ and $0.95 \AA)$ are larger than those of $\mathrm{Cu}^{+}(0.60$ and $0.77 \AA$ ), yet smaller than those of $\mathrm{Ag}^{+}$(1.00 and $1.15 \AA$ ), for cadmium (Cd) chalcogenides $\mathrm{Cu}^{+}$could be either a substitutional or an interstitial impurity, whereas $\mathrm{Ag}^{+}$will form primarily substitutional impurities. ${ }^{18,20,21}$ Also, the diffusion constant of $\mathrm{Ag}^{+}$is higher than that of $\mathrm{Cu}^{+} \cdot{ }^{20} \mathrm{Ag}^{+}$could therefore form an effective dopant in colloidal NCs, yet has, despite the recent successes in the doping of II-VI, ${ }^{5,14}$ III$\mathrm{V},{ }^{20,22}$ and $\mathrm{IV}-\mathrm{VI}^{23} \mathrm{NCs}$, not received the same attention so far as $\mathrm{Cu}$ and $\mathrm{Mn}$ dopants.

In general, two methods are employed to incorporate $\mathrm{Ag}^{+}$, either during synthesis of the host $\mathrm{NCs}^{24}$ or by post-synthesis cation exchange. ${ }^{18,20,21,25}$ The latter method separates doping from NC nucleation and growth, yielding more control over the doping level by the reaction time and the relative concentration of the added silver precursor. This strategy can also be employed in the opposite direction. Zhang et al. reported an inverted cation exchange reaction, mediated by ternary phosphines, to achieve CdS:Ag NCs, where they started with $\mathrm{Ag}_{2} \mathrm{~S} \mathrm{NCs}$ and replaced the $\mathrm{Ag}^{+}$by $\mathrm{Cd}^{2+} \cdot{ }^{26} \mathrm{~A}$ similar strategy also yielded CdSe:Ag QDs. ${ }^{19}$

With the recent development of a new class of colloidal NCs, 2D NPLs with uniform thickness and extended lateral dimensions, doping strategies can now be applied to these ultrathin semiconductor nanomaterials as well. ${ }^{7,27}$ CdSe NPLs possess a high photoluminescence quantum efficiency (PL QE). ${ }^{28-30}$ However, their emission typically displays a negligible Stokes shift, which can result in PL quenching in close-packed solid films. Therefore, CdSe:Ag NPLs could be 
Table 1. Ag Added to Synthesis, NPL Ag Concentration as Determined by ICP-OES, PL Peak Position $\left(\lambda_{\max }\right)$, fwhm and Average Lifetime $\left(\tau_{\mathrm{av}}\right)$ of the BE (First Row) and Ag Emission (Subsequent Rows), Overall Emission QE (PL QE) and Relative Contribution of BE and Ag Emission, for the Different CdSe:Ag NPLs

\begin{tabular}{ccccccc}
$\mathrm{Ag} / \mathrm{Cd}$ ratio added & $\mathrm{Ag} / \mathrm{Cd} / \mathrm{Se}($ atom \%) & $\lambda_{\max }(\mathrm{nm})$ & $\mathrm{fwhm}(\mathrm{meV})$ & $\tau_{\text {av }}(\mathrm{ns})$ & $\mathrm{PL}$ QE $(\%)$ & $\mathrm{BE} / \mathrm{Ag}$ PL ratio $(\%: \%)$ \\
$0: 1$ & $0: 58.3: 41.7$ & 509 & 43 & 5.3 & 51 & \\
$0.05: 1$ & $0.6: 60.7: 38.6$ & 650 & 401 & 252 & 63 & $65: 35$ \\
$0.1: 1$ & $0.9: 59.6: 39.6$ & 652 & 400 & 312 & 57 & $46: 54$ \\
$0.2: 1$ & $2.5: 58.9: 38.6$ & 665 & 413 & 406 & 54 & $12: 88$ \\
$0.4: 1$ & $4.7: 55.6: 39.8$ & 688 & 392 & 446 & 47 & $0.3: 99.7$ \\
$0.7: 1$ & $7.2: 49.6: 43.2$ & 730 & 403 & 459 & 56 & $0.07: 99.93$ \\
$1: 1$ & $8.8: 52.5: 38.7$ & 880 & 434 & 575 & & $0.05: 99.95$ \\
\hline
\end{tabular}

an excellent material for solid-state lighting ${ }^{11,12,14}$ or other applications that require strongly reduced self-absorption, such as LSCs. ${ }^{6,7,31}$ As an added advantage over colloidal QDs, the homogeneous thickness of NPLs results in a narrow emission spectrum, ${ }^{32,33}$ which should permit to spectrally separate BE and dopant contributions, whereas the peculiar shape enhances the absorption cross section compared to spherical QDs ${ }^{34}$ and yields anisotropic optical properties. ${ }^{35-37}$ Despite this promise, no example of Ag-doped NPLs has been reported to date.

Here, we demonstrate the synthesis of CdSe:Ag NPLs via post-synthesis cation exchange under tempered conditions in an ice bath, using silver acetate as the $\mathrm{Ag}$ precursor. Transmission electron microscopy (TEM) and X-ray diffraction (XRD) revealed that the doped NPLs maintain the shape, size, and crystal structure of the parent NPLs. The Ag concentration can be tuned by the relative concentration of $\mathrm{Ag}$ precursors used, and we reached a maximal $\mathrm{Ag}$ concentration of $8.8 \%$ in the doped NPLs. Ag doping results in an emission that can be tuned from 609 to $880 \mathrm{~nm}$, with a Stokes shift up to $372 \mathrm{~nm}(1 \mathrm{eV})$. This range is covered by varying the dopant concentration and by increasing the core thickness or growing $\mathrm{CdSe} / \mathrm{CdS}$ hetero-NPLs. The smallest dopant concentration leads to a PL QE as high as 63\%, and with an increased $\mathrm{Ag}$ concentration, the values remain around $45-55 \%$.

\section{RESULTS AND DISCUSSION}

CdSe NPLs with a thickness of 4.5 monolayers (MLs) were first synthesized following a previously published procedure. ${ }^{38}$ The purified NPLs were dispersed in hexane, and the concentration of NPLs was determined by the method reported by Achtstein et al. ${ }^{34}$ The $\mathrm{Cd}$ atomic concentration was calculated from the number of $\mathrm{CdSe}$ units present per NPL (for 4.5 ML NPLs, a $1.36 \mathrm{~nm}$ thickness was taken), multiplied with the concentration of NPLs. Ag dopants were introduced via cation exchange reaction. In an initial series of experiments, the CdSe NPLs were mixed with different silver salt solutions (silver nitrate, silver trifluoroacetate) following standard procedures for colloidal NCs. ${ }^{21,25}$ However, we observed that the NPLs were not stable under these conditions, likely because of the rapid cation exchange in the $1.36 \mathrm{~nm}$ thin NPLs (Supporting Information, Figure S1).

To avoid this, we resorted to silver acetate, which reacts more slowly, and we further reduced the reaction rate by performing the exchange around $0{ }^{\circ} \mathrm{C}$ in an ice bath (under ambient atmosphere, Figure 1a). In a typical reaction, we added silver acetate, in an $\mathrm{Ag} / \mathrm{Cd}$ molar ratio of $0.2: 1$, to a solution of CdSe NPLs under continuous stirring. The reaction was allowed to proceed for $30 \mathrm{~min}$. The absorbance and PL spectra of the resulting purified solution are presented in Figure 1b. After exchange, a broad peak with maximum at 665 $\mathrm{nm}$ appears in the $\mathrm{PL}$ spectrum next to the signature $\mathrm{BE}$ emission of the 4.5 ML NPLs at $509 \mathrm{~nm}$. Note that the absorbance spectrum of the CdSe:Ag NPLs yields no particular features in the region of the broad PL peak. The PL excitation spectra of the CdSe:Ag NPLs, collected at the BE $(509 \mathrm{~nm})$ and the dopant $(665 \mathrm{~nm})$ emission wavelength, respectively, are largely superposed (Figure 1c), demonstrating that both the $\mathrm{BE}$ and dopant emission originate from absorption in the NPLs and confirming the incorporation of $\mathrm{Ag}$ into the NPLs. Under UV illumination (inset of Figure 1c), we can appreciate the bright yellow emission of the CdSe:Ag NPLs, compared to the green-emitting original NPLs. The schematics of the multistep recombination process leading to the dopant $\mathrm{PL}$ is depicted in Figure 1d, showing, after photoexcitation (step 1, black arrow), the initial capture of the excited valence band (VB) hole in $\mathrm{Ag}^{+}$, following the reaction $\mathrm{Ag}^{+}+\mathrm{h}^{+} \rightarrow \mathrm{Ag}^{2+}$ (step 2, orange arrow), and the subsequent radiative decay of the $\mathrm{CB}$ electron into the dopant center (step 3, red arrow), resulting in a Stokes-shifted emission and a concomitant reduction of the metastable $\mathrm{Ag}^{2+}$ site to its stable +1 groundstate configuration. ${ }^{19}$

TEM images of the CdSe NPLs show a rectangular shape with an average length of $28 \pm 3 \mathrm{~nm}$ and a width of $3.7 \pm 0.5$ $\mathrm{nm}$ (Figure 1e). For the doped NPLs, we measured similar dimensions of $28 \pm 4 \mathrm{~nm}$ by $3.7 \pm 0.7 \mathrm{~nm}$ (Figure 1f), confirming that the cation exchange, performed under tempered conditions, does not modify the overall NPL size. High-resolution TEM (HRTEM) images of CdSe:Ag NPLs are presented in Figure 1g. Both the side- and top-view HRTEM images show that they are highly crystalline, with 3.4 and $2.1 \AA d$-spacings corresponding to the (111) and (220) planes of zinc-blende CdSe, comparable to other reported zincblende CdSe NPLs. ${ }^{30,39}$ The XRD patterns (Figure 1h) confirm the HRTEM results. As the diffraction peaks occur at the same angles for CdSe (green pattern) and CdSe:Ag (red pattern, see also Supporting Information, Table S1), we can again conclude that no significant changes are imposed on the overall crystal structure after doping.

The relative concentration of silver acetate affects the $\mathrm{Ag}$ doping concentration in the final CdSe:Ag NPLs. We performed cation exchange reactions, exposing the CdSe NPLs for $30 \mathrm{~min}$ to silver acetate in an ice bath, with $\mathrm{Ag} / \mathrm{Cd}$ molar ratios varying from $0.05: 1$ to $1: 1$, and we quantified the incorporation of $\mathrm{Ag}$ into the NPLs with inductively coupled plasma optical emission spectrometry (ICP-OES, Table 1). We observed a monotonic increase of the amount of $\mathrm{Ag}$ in the $\mathrm{CdSe}$ :Ag composition, eventually reaching $8.8 \%$. Even at these concentrations, no variation of the NPL size and shape was observed (Supporting Information, Figure S2). From the CdSe NPL dimensions, $28 \mathrm{~nm}$ by $3.7 \mathrm{~nm}$ and a thickness of $1.36 \mathrm{~nm}$, 
we estimated that the range of $0.6-8.8 \% \mathrm{Ag}$ corresponds to about $30-440$ atoms per NPL. Note that the ICP-OES data indicate that only about $10-15 \%$ of $\mathrm{Ag}$ present in solution is actually incorporated into the NPLs.

We also highlight that, at such high nominal doping levels, no undoped NPLs are statistically expected to be found in the ensemble. Therefore, the observation of residual BE PL in Figure $1 \mathrm{~b}$ indicates that only a subset of the $\mathrm{Ag}$ dopants diffuses inside the NPLs and actively participates in the excitonic decay, whereas the remaining fraction of the $\mathrm{Ag}$ cations likely adsorbs onto the particle surface (see also the schematic in Figure 1a). Support for this interpretation is provided by the comparison between the absorption spectra of the undoped and doped NPLs. Specifically, independent of the Ag concentration, the normalized CdSe:Ag NPL absorption profiles show spectral features that match well with the original CdSe NPLs (Figure 2a). Below the CdSe BE, Ag-doped NPLs
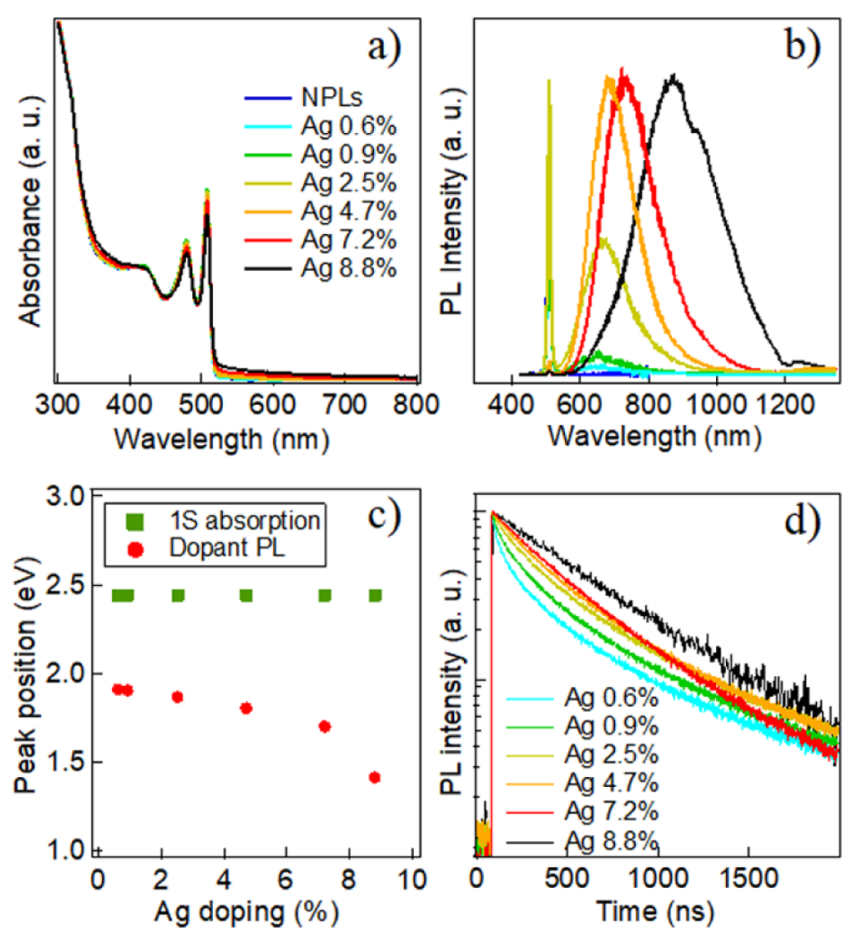

Figure 2. (a) UV-vis absorbance spectra of CdSe:Ag NPLs at increasing $\mathrm{Ag}$ concentration. (b) Corresponding steady-state PL spectra $\left(\lambda_{\mathrm{ex}}=400 \mathrm{~nm}\right.$, same color labels as the absorbance spectra). (c) Plot of the PL peak position against $\mathrm{Ag}$ concentration, highlighting the red shift of the dopant emission (red dots), whereas the $\mathrm{BE}$ absorption remains fixed (green squares). (d) PL decay traces of the dopant emission for different doping concentrations.

exhibit a measurable, yet very weak absorption tail that can be associated with the direct absorption in $\mathrm{Ag}^{+}$centers, consistent with the recent observation in CdSe:Ag QDs, ${ }^{19}$ where a clear absorption feature could be discerned. The weak subgap absorption intensity observed for our NPLs, despite the nominally comparable doping level, can be rationalized when considering the different preparation methods employed. In ref 19, CdSe:Ag NCs were obtained via an incomplete cation exchange reaction starting from $\mathrm{Ag}_{2} \mathrm{Se} \mathrm{NCs}$, which enables to retain a large amount of $\mathrm{Ag}$ cations in the interior of the doped NCs. Here, on the other hand, Ag doping was achieved by exposing CdSe NPLs to silver acetate. The observed differences between the spectral features of the two
Ag-doped CdSe nanostructures with comparable overall Ag concentration corroborate the picture that the current procedure leads to a larger fraction of surface-adsorbed Ag, and thus a lower concentration of $\mathrm{Ag}$ effectively incorporated into the CdSe lattice. This is also consistent with the previous results by Sahu et al. ${ }^{21}$ and Morgan and Kelley ${ }^{40}$ on CdSe QDs, Amit et al. ${ }^{22}$ on InAs, and Kroupa et al. ${ }^{23}$ on PbSe and with the spectroscopic and spectroelectrochemical (SEC) results reported in the following sections.

Looking closer at the absorption and PL spectra of doped NPLs as a function of the dopant concentration, we notice that the $\mathrm{BE}$ absorption peak remains fixed at $508 \mathrm{~nm}(2.44 \mathrm{eV}$, Figure 2a), whereas the spectral position of the dopant PL varies from 650 to $880 \mathrm{~nm}(1.41-1.91 \mathrm{eV}$, Figure $2 \mathrm{~b})$. The trends are quantified in Figure $2 \mathrm{c}$ as a function of the doping level. In the low-concentration limit, we obtained a Stokes shift of $0.53 \mathrm{eV}$, similar to the $0.4 \mathrm{eV}$ measured by Nelson et al. ${ }^{18}$ and Pinchetti et al. ${ }^{19}$ in CdSe QDs. At a higher concentration, the Stokes shift increases up to $1.03 \mathrm{eV}$. Although the dopant emission has a nearly constant full width at half-maximum (fwhm) of $0.39-0.43 \mathrm{eV}$ (Table 1), broadened by strong phonon coupling ${ }^{18}$ and a possible distribution of $\mathrm{Ag}$ concentrations across the NPL ensemble, an efficient spectral separation of the BE and dopant features is still obtained, assisted by the $9 \mathrm{~nm}(45 \mathrm{meV})$ narrow fwhm (calculated by doubling the half width of the red edge of the absorption peak) of the NPL BE absorption. The fixed spectral position of the latter confirms that the electronic structure of the host NPLs is not affected by the inclusion of Ag dopants, even at a high concentration. On the other hand, the shifting dopant emission suggests that, with increasing $\mathrm{Ag}$ concentration, changes occur to the local energy levels of the dopants as the host goes from nearly pure CdSe to $\mathrm{CdSe}: \mathrm{Ag}$ with $8.8 \% \mathrm{Ag}$, possibly because of the local crystal field modifications or small $\mathrm{Ag}_{2} \mathrm{Se}$ cluster formation. $^{20,24,41}$ For comparison, $2 \mathrm{~nm} \mathrm{Ag}_{2} \mathrm{Se}$ QDs show emission with a PL maximum at $1030 \mathrm{~nm}^{42}$

The Ag doping level is also found to modify the dynamics of the dopant-mediated PL. Specifically, the lightest doped CdSe:Ag NPLs exhibit a multiexponential decay (Figure 2d, Supporting Information Table S2) likely because of a distribution of decay pathways. Interestingly, upon increasing the doping level, this progressively turns into a singleexponential behavior that attests to an evolution toward a homogeneous doping of the CdSe:Ag NPL ensemble. As shown in Table 1, such a trend is accompanied by a gradual increase of the amplitude-averaged PL lifetime. For the heaviest doped NPLs, lifetimes are about a factor of 2 longer than that of CdSe:Ag QDs, which yielded 200 ns. ${ }^{18,19}$ Hence, the smaller subgap absorption tail observed in Figure $2 \mathrm{a}$ is, next to the lower effective concentration of $\mathrm{Ag}$ inside the lattice compared to CdSe:Ag QDs, also caused by the weak lateral confinement regime in NPLs, leading to an electron that is bound to the localized hole only by Coulomb interactions, which may yield a reduced electron-hole overlap compared to QDs.

Remarkably, all samples display a high absolute PL QE, up to $63 \%$ for the smallest dopant concentration and evolving toward values around $45-55 \%$ when the $\mathrm{Ag}$ concentration is increased (Table 1). These PL QEs are comparable to undoped CdSe NPLs despite the significantly longer PL lifetimes (Figure 2d, Table 1), suggesting that carrier localization on the Ag dopants and surface passivation by the adsorbed Ag ions (as further confirmed by the SEC analysis 
reported below) lead to a suppression of the nonradiative recombination rate. Focusing further on the broad dopant emission, its relative intensity scales with the $\mathrm{Ag}$ concentration (Figure 2b, Table 1). Starting at $4.7 \%$ Ag concentration, more than $99 \%$ of the emission originates from the Ag dopant level, whereas samples with a lower $\mathrm{Ag}$ concentration maintain a finite contribution of BE PL. This supports the assumption that $\mathrm{Ag}$ is initially adsorbed on the NPL surface, whereas only at higher concentration they also become substitutional dopants and act as deep hole acceptor states. ${ }^{18,40,41}$ The ratio of doped and undoped-or surface-decorated-NPLs can be quantified as follows. The initial CdSe NPLs have a PL QE of 51\%, similar to the PL QE of the CdSe:Ag NPLs that display only dopant emission (samples with 4.7 and $7.2 \% \mathrm{Ag}$, yielding an average PL QE of 51.5\%). When assuming that the PL QE of CdSe and CdSe:Ag NPLs does not vary significantly over the different samples, one can derive that the relative weight of the dopant emission directly represents the fraction of NPLs where Ag dopants are inserted into the CdSe lattice, and Table 1 hereby shows that the CdSe:Ag NPL samples with an $\mathrm{Ag}$ concentration below $4.7 \%$ maintain a distinct fraction of undoped NPLs in the ensemble. Nevertheless, the tunable PL spectra, combined with the minimal overlap between the absorption and PL spectra and the predominant contribution of the Ag dopant emission to the overall spectrum at larger $\mathrm{Ag}$ concentration, make CdSe:Ag NPLs attractive phosphors for optical imaging or down-conversion of blue or solar light in LEDs and LSCs, respectively. ${ }^{5,8,31}$

For a closer examination of the carrier dynamics in the CdSe:Ag NPLs, we investigated the hole relaxation process using transient absorption spectroscopy. As the CdSe:Ag NPLs have a nearly negligible Ag-related absorption band (Figure 2a), hole relaxation rates can no longer be derived directly from the buildup of $\mathrm{Ag}$ absorption, as was done previously on CdSe:Ag QDs. ${ }^{19}$ Instead, we inferred this from the $\mathrm{BE}$ absorption using state-resolved transient absorption spectroscopy. This method has already been applied successfully to study carrier cooling, exciton-phonon coupling, and multiexciton interactions. ${ }^{43}$

Here, we excited the CdSe and CdSe:Ag NPLs at a wavelength resonant with the heavy hole exciton (HH-X) transition and monitored the bleach signal of the heavy hole (dark blue area) and light hole exciton (LH-X, light blue area) transitions (see Figure 3a,b). Importantly, both excitons are derived from the same $\mathrm{CB}$ state but from different VB states. Hence, the hole capture from the $\mathrm{HH}$ exciton will leave behind a single $\mathrm{CB}$ electron that will affect light absorption by both the HH-X and LH-X transitions. Electron capture, on the other hand, will leave behind a heavy hole, which should only affect light absorption by the HH-X transition. Experimentally, we found that the HH-X and LH-X bleach traces exhibit the same decay dynamics at longer delay times. This indicates that the $\mathrm{HH}$ exciton suffers from rapid hole capture, after which the decay of the remaining $\mathrm{CB}$ electron determines the bleach of the HH-X and LH-X absorbance. Consequently, when normalizing both traces at a long delay time (Figure $3 \mathrm{c}$ ), and subtracting the former from the latter, one obtains the hole dynamics of the system (Figure $3 \mathrm{~d}$ ).

Fitting the resulting trace for CdSe NPLs (black curve) yields three time constants (Supporting Information, Table S3), reflecting a range of surface traps and associated decay constants, as for CdSe QDs. ${ }^{44}$ The amplitude-averaged decay constant equals 23.5 ps. When constructing and fitting similar
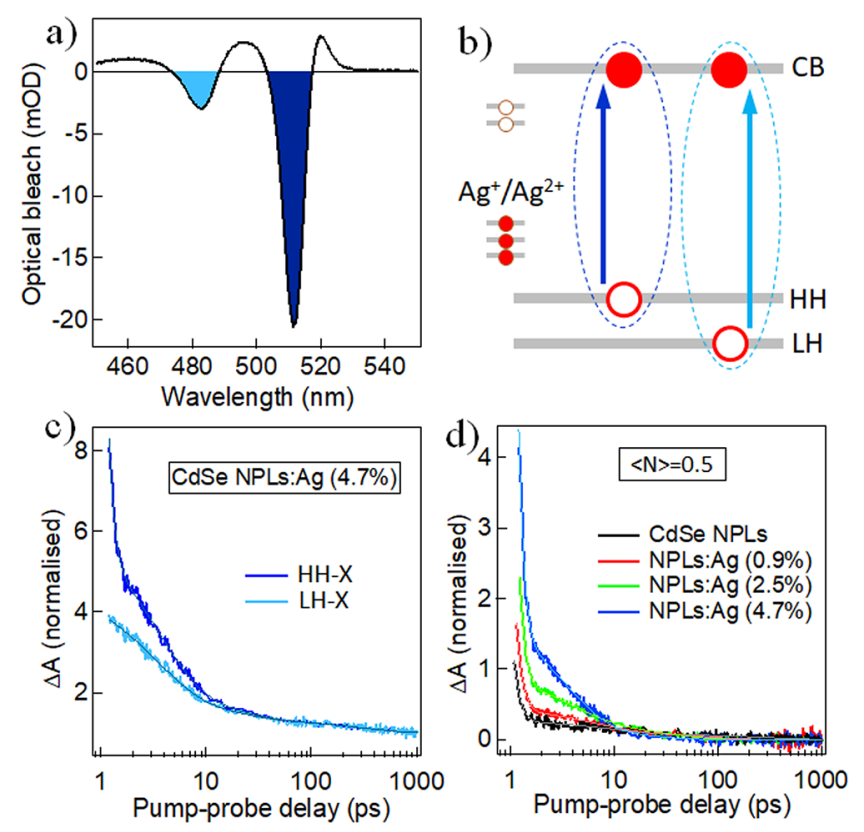

Figure 3. (a) Identification of the HH-X (dark blue) and LH-X (light blue) bands in the transient absorption spectra, which were used for the analysis of the hole dynamics. (b) Schematics illustrating the band structure of CdSe:Ag NPLs, including occupied and unoccupied surface states. (c) HH-X (dark blue) and LH-X (light blue) transient absorption traces (shown for the sample with $4.7 \% \mathrm{Ag}$ ). (d) Hole dynamics ( $\mathrm{HH}$ trace subtracted with $\mathrm{LH}$ trace) for CdSe NPLs (black) and CdSe:Ag NPLs with 0.9\% (red), 2.5\% (green), and 4.7\% (blue) Ag.

traces for CdSe:Ag NPLs with the Ag concentration of 0.9, 2.5, and $4.7 \%$, respectively (Figure $3 \mathrm{~d}$ ), we observed a progressive decrease of the relaxation constant, from 6.5 ps down to 1.9 ps for the sample with the highest Ag concentration (where also $99 \%$ of the emission originates from the dopants). As the decay constant is significantly smaller than that for undoped CdSe, and similar to the one found in CdSe:Ag QDs, ${ }^{19}$ we can ascribe it to hole relaxation into the $\mathrm{Ag}$ dopant. Note that the ultrafast relaxation should lead to a complete quenching of $\mathrm{BE}$ $\mathrm{PL}$, again corroborating that, at low Ag concentrations, we have a heterogeneous NPL ensemble, and only when exposing it to sufficiently high concentrations of Ag precursors, all NPLs are Ag-doped.

The ultrafast decay into the $\mathrm{Ag}^{+}$dopant level together with the surface passivation effect posited by the surface-adsorbed $\mathrm{Ag}$ atoms also modifies the interaction of charge carriers with surface defects. To shed light onto this mechanism and to clarify the nature of the intragap dopant state, we performed SEC experiments on CdSe:Ag NPLs with an Ag concentration of $2.5 \%$. In these experiments, the NPLs are excited with a UV laser, and PL is collected while the position of the Fermi level $(\mathrm{FL})$ is tuned by the application of an EC potential $\left(V_{\mathrm{EC}}\right)$. Specifically, a negative $V_{\mathrm{EC}}$ corresponds to the raising of the FL and leads to passivation of electron-poor surface states acting as nonradiative traps for the photogenerated $\mathrm{CB}$ electrons. Concomitantly, the excess electrons accumulated in the surface defects become efficient traps for the photogenerated VB holes. Conversely, a positive $V_{\mathrm{EC}}$ (corresponding to lowering the FL) depletes the NPL of the photoexcited electrons and concomitantly passivates the electron-rich defect states acting as efficient hole traps. The effect of $V_{\mathrm{EC}}$ on the emission efficiency of the NPLs is therefore determined by the 

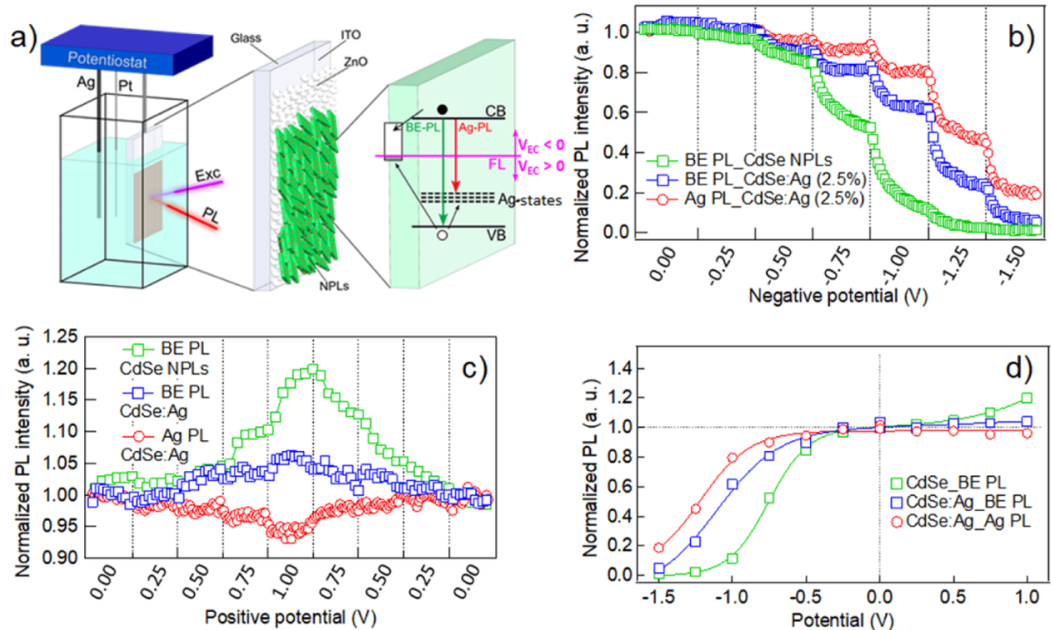

Figure 4. (a) Schematic depiction of the custom SEC setup consisting of an EC cell with $\mathrm{TBAClO}_{4}$ in propylene carbonate (0.1 M) as an electrolyte and a working electrode comprising of an ITO-coated glass slide covered with a layer of ZnO nanoparticles (NPs) and CdSe:Ag NPLs. Also shown is the radiative recombination pathway (red arrow) of photoexcited CB electrons to the intragap Ag state following the capture of the VB hole (black arrow). The green arrow indicates the BE radiative recombination. Competitive nonradiative carrier trapping processes to defect states are shown by black arrows. FL is indicated by a pink line. (b-c) SEC measurements on CdSe and CdSe:Ag NPLs at negative (b) and positive (c) bias $V_{\mathrm{EC}}$. The evolution of the PL intensity after each potential step indicates a slow charging/decharging of the NPL film. (d) PL intensity as a function of $V_{\mathrm{EC}}$ for CdSe NPLs (green squares), and the BE emission (blue squares) and Ag emission (red circles) of CdSe:Ag NPLs. Data were taken from the final values in panels $(b, c)$, just before a subsequent potential step was applied.
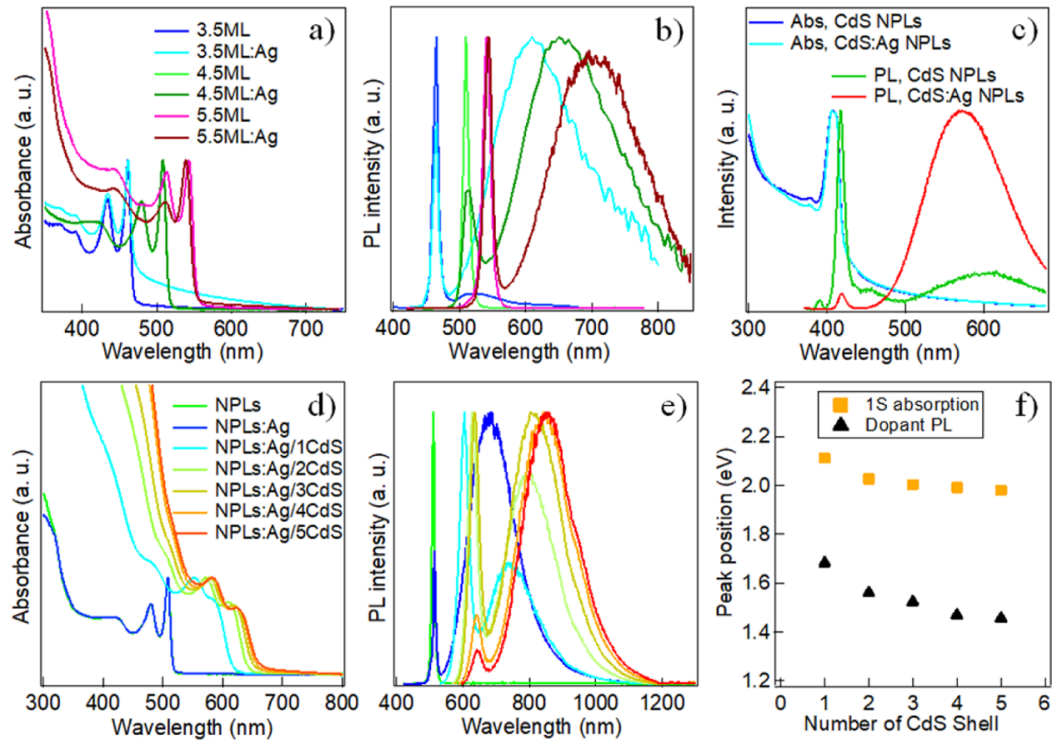

Figure 5. (a) Absorption and (b) PL spectra of CdSe and CdSe:Ag NPLs with thicknesses of 3.5, 4.5, and 5.5 MLs. (c) Absorption and PL spectra of CdS and CdS:Ag NPLs. (d) Absorption and (e) PL spectra of 4.5 ML CdSe:Ag NPLs and the corresponding CdSe:Ag/CdS core-shell NPLs with different number of CdS MLs. (f) Change of the BE absorption and dopant PL peak positions with the number of CdS shell layers.

competition between the selective passivation/activation of carrier traps and their respective trapping rates and by the occupancy of the surface states at $V_{\mathrm{EC}}=0 \mathrm{~V} .^{45,46}$ For this reason, SEC measurements are an effective tool to determine the trapping mechanisms affecting the exciton decay. In the specific case of CdSe:Ag NPLs, the selectivity of SEC measurements for the $\mathrm{BE}$ carriers of different signs further allows us to probe the electronic nature of the intragap Ag state responsible for the observed photophysics. The custom SEC experimental setup is depicted in Figure 4a. Here, a working electrode composed of a thin film of NPLs dip-coated onto a $\mathrm{ZnO}$-coated indium tin oxide (ITO) electrode is immersed into an electrolytic solution of tetrabutylammonium $(0.1 \mathrm{M})$ in propylene carbonate. Figure 4a also depicts the band energy diagram of CdSe:Ag NPLs. According to a previous report, ${ }^{19}$ the $\mathrm{FL}$ at $V_{\mathrm{EC}}=0 \mathrm{~V}$ is placed between the intragap states of $\mathrm{Ag}^{+}$dopants because of the fully occupied $d$-orbitals (electronic configuration $4 d^{10}$ ) and the host CB. Looking first at the undoped CdSe NPLs under a negative $V_{\mathrm{EC}}$, we observe an almost complete PL quenching (Figure 4b). In contrast, the application of a positive EC potential leads to a PL brightening of $\sim 20 \%$ (Figure $4 \mathrm{c}$ ). In agreement with a previous report, ${ }^{45}$ these observations indicate that the major nonradiative recombination channel in undoped CdSe NPLs is hole trapping. 
Moving on to Ag-doped NPLs (2.5\% Ag), Figure 4b,c reports the evolution of both the $\mathrm{BE}$ and the Ag-related emission intensities as a function of $V_{\mathrm{EC}}$. Upon increasing the negative $V_{\mathrm{EC}}$, we observe significant quenching of both emissions, although weaker compared to the undoped sample. Remarkably, at positive $V_{\mathrm{EC}}$, the $\mathrm{BE}$ and Ag-related recombination channels show opposite trends: $\mathrm{BE}$ shows progressive brightening with increasing $V_{\mathrm{EC}}$ similar to the undoped sample, whereas Ag PL is quenched by 5\% (Figure $4 c$ ). As the optical response of BE PL (arising from the subset of CdSe NPLs that only contain surface-adsorbed $\mathrm{Ag}$ ) to the application of both negative and positive $V_{\mathrm{EC}}$ is less pronounced than that for the sample that was not exposed to $\mathrm{Ag}$ (Figure 4d), the SEC experiments corroborate the picture that some nonradiative surface hole traps in the doped NPLs are passivated by surface-adsorbed $\mathrm{Ag}$ ions. It is also worth noting that, despite the longer PL lifetime, in doped NPLs, the Ag emission is less affected by the negative EC potential than the $\mathrm{BE}$ emission ( $\sim 95 \%$ vs $\sim 80 \%$ quenching respectively, Figure $4 \mathrm{~d}$ ), suggesting that the holes localized at the intragap Ag states are efficiently shielded from the surface traps. This is also in agreement with the picture of $\mathrm{Ag}$ serving as a hole acceptor with its $d$-states lying close to the $\mathrm{VB}$ of $\mathrm{CdSe}$, therefore introducing a competitive channel for the photogenerated holes to localization at the surface traps and to BE recombination.

Finally, to demonstrate that Ag doping of colloidal NPLs via cation exchange is a versatile method, we applied the same procedure to different core thicknesses and NPL compositions. First, 3.5 and 5.5 ML CdSe NPLs were prepared by standard protocols, ${ }^{47}$ and we performed a cation exchange reaction (with $\mathrm{Ag} / \mathrm{Cd}$ ratio of $0.2: 1$ ) as described above. The absorption and PL spectra of the CdSe and CdSe:Ag NPLs are presented in Figure 5a,b. The PL spectra indicate that the dopant PL can be tuned from 609 to $702 \mathrm{~nm}$ by increasing the thickness of the host NPLs from 3.5 to $5.5 \mathrm{ML}$. In contrast with the result obtained when varying the dopant concentration (Figure 2), here the red shift is controlled by a reduced quantum confinement of the (delocalized) first electron state, as evidenced by the similar shift of both BE absorption and dopant emission (Supporting Information, Figure S3). The procedure is also applicable to $\mathrm{CdS} \mathrm{NPLs}^{48}$ (Figure $5 \mathrm{c}$ ). $\mathrm{Ag}$ doping of 4.5 ML CdS NPLs - that emit at $417 \mathrm{~nm}$ - leads to a broad PL band centered at $570 \mathrm{~nm}$. Further tuning of the dopant emission can be achieved by growing a CdS shell around the CdSe:Ag NPLs, which we obtained with colloidal atomic layer deposition. ${ }^{49}$ Changes in the absorption and PL spectra of 4.5 ML CdSe:Ag NPLs (2.5\% Ag) are presented in Figure 5d,e. One ML of CdS shell already shifts the dopant emission to the near-infrared region, at $737 \mathrm{~nm}$. Finally, four more additional layers yielded emission at $852 \mathrm{~nm}$. The red shift of the dopant level can again be ascribed to the reduced confinement of the first electron state (Figure $5 \mathrm{f}$ ).

\section{CONCLUSIONS}

We have prepared colloidal Ag-doped CdSe NPLs by partial cation exchange. At an $\mathrm{Ag}$ concentration of $4.7 \%$ or higher, a uniform doping is achieved, as shown by the absence of residual $\mathrm{BE}$ emission and the single exponential decay of the dopant emission. The SEC and transient absorption measurements reveal that $\mathrm{Ag}^{+}$acts as an acceptor dopant, with a hole trapping rate of 1.9-6.5 ps, depending on the doping concentration. The dopant PL can be tuned by changing either the $\mathrm{Ag}$ concentration or by modifying the electron confinement energy by changing the thickness of the host NPLs or by coating CdSe:Ag NPLs with a CdS shell. With a tunable emission ranging from orange $(609 \mathrm{~nm})$ to nearinfrared $(880 \mathrm{~nm})$ wavelengths, large Stokes shifts resulting in a suppressed emission reabsorption, and PL QE values that exceed $50 \%$ and remain as high as the host NPLs, these fluorescent CdSe:Ag NPLs form an interesting addition to the class of colloidal 2D NC emitters, with potential applications in LSCs or light-emitting devices, where efficient emission needs to be combined with high transparency.

\section{EXPERIMENTAL METHODS}

Synthesis Materials. Cadmium nitrate tetrahydrate (99.997\%), sodium myristate ( $\geq 99 \%$ ), methanol (99.9\%), octadecene (ODE; $90 \%)$, cadmium(II) acetate $\left(\mathrm{Cd}(\mathrm{OAc})_{2} ; 99.995 \%\right)$, cadmium acetate dihydrate $\left(\mathrm{Cd}(\mathrm{OAc})_{2} \cdot 2 \mathrm{H}_{2} \mathrm{O}\right)$, oleic acid $(90 \%)$, trioctylphosphine (TOP; 90\%), hexane ( $\geq 95 \%)$, silver acetate (99.99\%), N-methylformamide (NMF, 99\%), acetonitrile (99.8\%), toluene (99.8\%), and ethanol $(\geq 99.8 \%)$ were purchased from Sigma-Aldrich. Cadmium oxide (99.999\%) and selenium powder (Se; 99.99\%) were purchased from Strem Chemicals. Ammonium sulfide (20-24\% aqueous solution) was purchased from Alfa Aesar. All chemicals were used without further purification. Silver acetate and Se were stored in a glovebox.

Preparation of Cadmium Myristate. Cadmium nitrate tetrahydrate $(3 \mathrm{~g})$ was dissolved in $200 \mathrm{~mL}$ of methanol. In another roundbottom flask, $5 \mathrm{~g}$ of sodium myristate was dissolved in $500 \mathrm{~mL}$ of methanol using sonication. The cadmium solution was then added dropwise to the myristate solution and stirred for $2 \mathrm{~h}$ at room temperature. The resulting precipitate of cadmium myristate was filtered and washed several times with methanol and was dried in a freeze dryer overnight.

Synthesis of 3.5 ML CdSe NPLs. ${ }^{47}$ In a three-neck flask, $240 \mathrm{mg}$ of $\mathrm{Cd}(\mathrm{OAc})_{2}, 150 \mu \mathrm{L}$ of oleic acid, and $15 \mathrm{~mL}$ of ODE were degassed for over $1 \mathrm{~h}$ at $80^{\circ} \mathrm{C}$. Then, under argon flow, the flask was heated to $180{ }^{\circ} \mathrm{C}$, and $150 \mu \mathrm{L}$ of $1 \mathrm{M}$ TOPSe was quickly injected. The reaction was stopped after $20 \mathrm{~min}$, and $2 \mathrm{~mL}$ of oleic acid was added when the reaction was cooled to room temperature. Finally, the NPLs were centrifuged twice at $6000 \mathrm{rpm}$ for $10 \mathrm{~min}$ with ethanol and were dispersed in hexane.

Synthesis of 4.5 ML CdSe NPLs. ${ }^{38}$ In a three-neck flask, $180 \mathrm{mg}$ of cadmium myristate, $30 \mathrm{mg}$ of $\mathrm{Se}$, and $15 \mathrm{~mL}$ of ODE were degassed for $1 \mathrm{~h}$ at $120^{\circ} \mathrm{C}$. Then, the flask was heated to $240{ }^{\circ} \mathrm{C}$ under argon flow. When the temperature reached $210^{\circ} \mathrm{C}, 80 \mathrm{mg}$ of $\mathrm{Cd}(\mathrm{OAc})_{2}$ was quickly introduced. The solution was held at $240{ }^{\circ} \mathrm{C}$ for $8 \mathrm{~min}$, and the reaction was then stopped. The temperature was reduced to 160 ${ }^{\circ} \mathrm{C}$, and $2 \mathrm{~mL}$ of oleic acid was added. When the temperature of the reaction solution reached room temperature, $15 \mathrm{~mL}$ of hexane was added. The mixture was then centrifuged at $3000 \mathrm{rpm}$ for $10 \mathrm{~min}$. The precipitate containing the mixture of 3.5 and 4.5 ML NPLs was suspended in $30 \mathrm{~mL}$ of hexane. The resultant solution was centrifuged at $6000 \mathrm{rpm}$ for $10 \mathrm{~min}$ to remove the $3.5 \mathrm{ML}$ NPLs as the precipitant.

Synthesis of 5.5 ML CdSe NPLs. ${ }^{47}$ In a three-neck flask, $170 \mathrm{mg}$ of cadmium myristate and $15 \mathrm{~mL}$ of ODE were degassed for $1 \mathrm{~h}$ at room temperature. Then, under argon flow, the flask was heated to $245^{\circ} \mathrm{C}$, and $1 \mathrm{~mL}$ of $0.15 \mathrm{M} \mathrm{Se}-\mathrm{ODE}$ (prepared by dissolving $12 \mathrm{mg}$ of Se in $1 \mathrm{~mL}$ of ODE by sonication) was injected. After $30 \mathrm{~s}$ of reaction, $90 \mathrm{mg}$ of $\mathrm{Cd}(\mathrm{OAc})_{2}$ was introduced. The solution was held at $245^{\circ} \mathrm{C}$ for $10 \mathrm{~min}$, and the temperature was then reduced to arrest the growth. At $150{ }^{\circ} \mathrm{C}, 2 \mathrm{~mL}$ of oleic acid was added, followed by the addition of $15 \mathrm{~mL}$ of hexane at room temperature. The mixture was then centrifuged at 3000 for $10 \mathrm{~min}$, and the precipitate containing the NPLs was suspended in $30 \mathrm{~mL}$ of hexane. To remove the byproducts (3.5 and 4.5 ML CdSe NPLs), few drops of acetonitrile were added and centrifuged at $6000 \mathrm{rpm}$ for $30 \mathrm{~min}$. The final decant contains the 5.5 ML CdSe NPLs. 
Silver Doping of CdSe NPLs. Taking the example of $4.5 \mathrm{ML}$ NPLs, the concentration of cadmium ions in the NPL stock solution was calculated first by using the intrinsic absorption coefficient reported by Achtstein et al. ${ }^{33}$ for $4.5 \mathrm{ML}$ NPLs and the NPL dimensions as determined by TEM. The silver stock solution was prepared by dispersing silver acetate in hexane $(1 \mathrm{mg} / \mathrm{mL})$ in a glovebox. In a fixed amount of NPL solution, different volumes of silver stock solution were added to achieve different dopant concentrations. The reactions were performed in an ice bath for 30 min, under stirring in ambient atmosphere. Afterward, the unreacted silver salts were removed by centrifuging the suspension at $3000 \mathrm{rpm}$ for $5 \mathrm{~min}$. To dope other NPLs, the same procedure was adopted.

Synthesis of CdSe:Ag/CdS Core/shell NPLs. The NPLs were synthesized following the colloidal atomic layer deposition approach. ${ }^{49}$ The first layer of sulfur was introduced by transferring the CdSe:Ag NPLs from hexane to $3 \mathrm{~mL}$ of NMF, to which $100 \mu \mathrm{L}$ of ammonium sulfide ( $20 \%$ in water) was added. Afterward, the polar solution was isolated. A mixture of acetonitrile and toluene (1:4) was added to precipitate the NPLs. After centrifugation at $6000 \mathrm{rpm}$ for $10 \mathrm{~min}$, they were dispersed in $3 \mathrm{~mL}$ of NMF. This was repeated at least two times to remove the sulfur precursor from the solution. To grow the first layer of cadmium, $3 \mathrm{~mL}$ of $0.5 \mathrm{M}$ cadmium acetate in NMF was introduced, and the suspension was stirred for $1 \mathrm{~h}$. The NPLs were again purified as described above and dispersed in $3 \mathrm{~mL}$ of NMF. For thicker shells, the same procedure was repeated until the desirable thickness was reached. The final CdSe:Ag/CdS core/shell NPLs were dispersed in $3 \mathrm{~mL}$ of toluene with the addition of $250 \mu \mathrm{L}$ of oleic acid to stabilize them in solution. The excess oleic acid was removed by precipitation of the NPLs with acetonitrile, followed by resuspension in hexane.

Synthesis of 4.5 ML CdS NPLs. The CdS NPLs were synthesized following the method of $\mathrm{Li}$ et al. ${ }^{48}$ In a three-neck flask, $53 \mathrm{mg}$ of cadmium acetate dehydrate, $1 \mathrm{~mL}$ of $\mathrm{S}-\mathrm{ODE}$ solution $(1.6 \mathrm{mg} \mathrm{S}$ per $\mathrm{mL}$ of ODE), $0.063 \mathrm{~mL}$ of oleic acid, and $5 \mathrm{~mL}$ of ODE were degassed with $\mathrm{Ar}$ at room temperature for $15 \mathrm{~min}$, then heated to 260 ${ }^{\circ} \mathrm{C}$ under Ar flow, and kept under $260{ }^{\circ} \mathrm{C}$ for $1 \mathrm{~min}$. Afterward, the reaction flask was slowly cooled, and at $160^{\circ} \mathrm{C}, 1 \mathrm{~mL}$ of oleic acid was added. An excess of hexane was added, and the suspension was centrifuged at $6000 \mathrm{rpm}$ for $10 \mathrm{~min}$. The precipitate was suspended in hexane.

Elemental Analysis. For ICP-OES measurements, the doped NPLs were precipitated by adding excess acetonitrile and centrifuging at $6000 \mathrm{rpm}$ for $10 \mathrm{~min}$, and finally they were suspended in hexane. ICP-OES measurements were performed on an aiCAP 6000 spectrometer (Thermo Scientific). The NPLs were digested in aqua regia overnight prior to the measurements.

Transmission Electron Microscopy. The samples were purified once more by adding excess acetonitrile, centrifuging at $6000 \mathrm{rpm}$ for $10 \mathrm{~min}$, and suspending in hexane, followed by drop-casting a dilute suspension onto carbon-coated copper grids. TEM images were acquired on a JEOL JEM-1011 microscope equipped with a thermionic gun operated at an accelerating voltage of $100 \mathrm{kV}$. HRTEM measurements were carried out on a JEOL JEM-2200FS microscope equipped with a Schottky emitter operated at $200 \mathrm{kV}$, a CEOS spherical aberration corrector for the objective lens, and an incolumn energy filter (Omega-type).

XRD Measurements. The XRD patterns were measured on a PANanalytical Empyrean X-ray diffractometer equipped with a 1.8 $\mathrm{kW} \mathrm{Cu} \mathrm{K} \alpha$ ceramic X-ray tube and a PIXcel ${ }^{3 \mathrm{D}} 2 \times 2$ area detector operating at $45 \mathrm{kV}$ and $40 \mathrm{~mA}$. Samples for the XRD measurements were prepared by drop-casting a concentrated NPL dispersion onto a miscut silicon substrate. The diffraction patterns were collected using a parallel-beam geometry and symmetric reflection mode.

Optical Characterization. Absorbance spectra of the NPLs in hexane were recorded using a Varian Cary 5000 UV-vis-NIR spectrophotometer. Steady-state and time-resolved PL spectra were measured using an Edinburgh Instruments FLS920 spectrofluorometer. The steady-state PL was collected by exciting the samples at 400 $\mathrm{nm}$ with a xenon lamp. The same setup coupled to an integrating sphere was used for PL QE measurements. The PL decay traces were recorded by exciting the samples at $405 \mathrm{~nm}$ using a 50 ps laser diode at a repetition rate of $0.05-1 \mathrm{MHz}$.

Transient Absorption Spectroscopy. The samples were excited using $110 \mathrm{fs}$ pump pulses at the spectral position of the HH-X transition $(510 \mathrm{~nm})$, which were created from the $800 \mathrm{~nm}$ fundamental (Spitfire Ace, Spectra Physics) through a nonlinear conversion in an optical parametric amplifier (Light Conversion TOPAS). Equally short probe pulses were generated in a thin $\mathrm{CaF}_{2}$ crystal using the $800 \mathrm{~nm}$ fundamental. The pulses were delayed relative to the pump using a mechanical delay stage. The probe spectrum in our experiments covered the UV-vis window from 350 $\mathrm{nm}$ up to $750 \mathrm{~nm}$; yet, we focused our attention on the region near the $\mathrm{BE}$, that is, the HH-X and LH-X transitions. To calculate the average number of absorbed photons $\langle N\rangle$ per platelet, we used the relation: $\langle N\rangle=J_{\mathrm{ph}} \times \sigma$, where $\sigma$ is the absorption cross section and $J_{\mathrm{ph}}$ is the photon flux. The flux was calculated from the average excitation power $(0.1 \mathrm{~mW})$, the repetition rate $(500 \mathrm{~Hz})$, and the beam area $\left(0.029 \mathrm{~cm}^{2}\right)$. The cross section was calculated as $3.0 \times$ $10^{-14} \mathrm{~cm}^{2}$ at $510 \mathrm{~nm}$, using the method of Achtstein et al., ${ }^{34}$ taking the specific NPL dimensions into account. The data shown was obtained at $\langle N\rangle=0.5$.

Spectroelectrochemistry. ITO-coated glass slides $(50 \times 7 \times 0.7$ $\mathrm{mm}, R_{\mathrm{S}}<100 \Omega$ ) were purchased from Delta Technologies (part no. CG-90IN-CUV). The ITO-coated surface was first covered with zinc oxide $(\mathrm{ZnO})$ NPs (Nanograde, $\sim 50 \mathrm{~nm}$ diameter) to avoid NPL emission quenching by fast charge/energy transfer to ITO. The $\mathrm{ZnO}$ NP layer ( $\sim 60 \mathrm{~nm}$ thick, as measured using a Dektak profilometer) was deposited by dip coating the glass/ITO substrate into an ethanol suspension of $\mathrm{ZnO}$ NPs $(2 \mathrm{mg} / \mathrm{mL}$, one dip for $10 \mathrm{~s})$ and annealing at $150{ }^{\circ} \mathrm{C}$ for $10 \mathrm{~min}$ in a nitrogen glovebox. To test the stability of the glass/ITO/ZnO NP substrates during potential scans, we performed control experiments in which we monitored the changes in the optical absorption spectra for prolonged exposures to negative and positive potentials. The results of these measurements indicate that the substrates are unaffected by either positive or negative EC potentials for the exposure times of tens of minutes, which are much longer than the measurement time used in our SEC experiments $(\sim 10 \mathrm{~min})$. The NPLs were deposited onto the ZnO NP layer as a film of few MLs by dip coating from a dilute hexane solution. The ITO was connected as a working electrode to the potentiostat (BioLogic SP-200 research grade potentiostat/galvanostat), and the film was placed into a quartz cuvette filled with the electrolyte $(0.1 \mathrm{M}$ tetrabutylammonium perchlorate in propylene carbonate). Silver and platinum wires were used as the quasi-reference and counter electrodes, respectively. All potentials reported in this work are measured relative to the quasireference silver electrode during the staircase voltammetry scans ( 360 $\mathrm{s}$ per scan, $60 \mathrm{~s}$ per potential step). The film was excited at $3.06 \mathrm{eV}$ with an EPL 405 (Edinburgh Inst.) 40 ps pulsed diode laser, and the emitted light was collected with a focusing lens and sent to a spectrometer coupled to a TM-C10083CA Hamamatsu minispectrometer.

\section{ASSOCIATED CONTENT}

\section{Supporting Information}

The Supporting Information is available free of charge on the ACS Publications website at DOI: 10.1021/acs.chemmater.8b05334.

Experimental methods, additional TEM images, XRD peak values and lattice constants, decay components of time-resolved fluorescence and transient absorption traces, and additional data for CdSe:Ag NPLs with different thicknesses (PDF)

\section{AUTHOR INFORMATION}

\section{Corresponding Author}

*E-mail: iwan.moreels@ugent.be. 


\section{ORCID $\odot$}

Ali Hossain Khan: 0000-0001-7155-0200

Valerio Pinchetti: 0000-0003-3792-3661

Zeger Hens: 0000-0002-7041-3375

Pieter Geiregat: 0000-0001-7217-8738

Sergio Brovelli: 0000-0002-5993-855X

Iwan Moreels: 0000-0003-3998-7618

\section{Author Contributions}

The manuscript was written through contributions of all authors. All authors have given approval to the final version of the manuscript.

Notes

The authors declare no competing financial interest.

\section{ACKNOWLEDGMENTS}

Z.H., D.V.T., and I.T. acknowledge the Research Foundation Flanders (project 17006602). P.G. acknowledges the FWOVlaanderen for a Postdoctoral Fellowship. I.M. and Z.H. acknowledge Ghent University for funding (GOA 01G01019). This project has also received funding from the European Union's Horizon 2020 research and innovation program (grant agreement no. 696656 GrapheneCore1) and the European Research Council (ERC, grant agreement no. 714876 PHOCONA). V.P. and S.B. are grateful to the MIUR "Dipartimenti di Eccellenza 2017 Project-Materials for Energy".

\section{REFERENCES}

(1) Pietryga, J. M.; et al. Spectroscopic and Device Aspects of Nanocrystal Quantum Dots. Chem. Rev. 2016, 116, 10513-10622.

(2) Kovalenko, M. V.; et al. Prospects of Nanoscience with Nanocrystals. ACS Nano 2015, 9, 1012-1057.

(3) Talapin, D. V.; Lee, J.-S.; Kovalenko, M. V.; Shevchenko, E. V. Prospects of Colloidal Nanocrystals for Electronic and Optoelectronic Applications. Chem. Rev. 2010, 110, 389-458.

(4) Li, Q.; Lian, T. Area- and Thickness-Dependent Biexciton Auger Recombination in Colloidal CdSe Nanoplatelets: Breaking the "Universal Volume Scaling Law". Nano Lett. 2017, 17, 3152-3158.

(5) Knowles, K. E.; et al. Luminescent Colloidal Semiconductor Nanocrystals Containing Copper: Synthesis, Photophysics, and Applications. Chem. Rev. 2016, 116, 10820-10851.

(6) Bradshaw, L. R.; Knowles, K. E.; McDowall, S.; Gamelin, D. R. Nanocrystals for Luminescent Solar Concentrators. Nano Lett. 2015 $15,1315-1323$.

(7) Sharma, M.; et al. Near-Unity Emitting Copper-Doped Colloidal Semiconductor Quantum Wells for Luminescent Solar Concentrators. Adv. Mater. 2017, 29, 1700821.

(8) Wu, P.; Yan, X.-P. Doped Quantum Dots for Chemo/Biosensing and Bioimaging. Chem. Soc. Rev. 2013, 42, 5489-5521.

(9) Santra, P. K.; Kamat, P. V. Mn-Doped Quantum Dot Sensitized Solar Cells: a Strategy to Boost Efficiency over 5\%. J. Am. Chem. Soc. 2012, 134, 2508-2511.

(10) Tenne, R.; et al. From Dilute Isovalent Substitution to Alloying in CdSeTe Nanoplatelets. Phys. Chem. Chem. Phys. 2016, 18, 1529515303 .

(11) Khan, A. H.; et al. Efficient Solid-State Light-Emitting CuCdS Nanocrystals Synthesized in Air. Angew. Chem., Int. Ed. 2015, 54, 2643-2648.

(12) Zhang, Z.; et al. Dual Emissive $\mathrm{Cu}: \mathrm{InP} / \mathrm{ZnS} / \mathrm{InP} / \mathrm{ZnS}$ Nanocrystals: Single-Source "Greener" Emitters with Flexibly Tunable Emission from Visible to Near-Infrared and Their Application in White Light-Emitting Diodes. Chem. Mater. 2015, 27, 1405-1411.

(13) Avidan, A.; Oron, D. Large Blue Shift of the Biexciton State in Tellurium Doped CdSe Colloidal Quantum Dots. Nano Lett. 2008, 8, 2384-2387.
(14) Pradhan, N.; Sarma, D. D. Advances in Light-Emitting Doped Semiconductor Nanocrystals. J. Phys. Chem. Lett. 2011, 2, 28182826.

(15) Meinardi, F.; et al. Doped Halide Perovskite Nanocrystals for Reabsorption-Free Luminescent Solar Concentrators. ACS Energy Lett. 2017, 2, 2368-2377.

(16) Hazarika, A.; Pandey, A.; Sarma, D. D. Rainbow Emission from an Atomic Transition in Doped Quantum Dots. J. Phys. Chem. Lett. 2014, 5, 2208-2213.

(17) Pradhan, N.; Peng, X. Efficient and Color-Tunable Mn-Doped ZnSe Nanocrystal Emitters: Control of Optical Performance via Greener Synthetic Chemistry. J. Am. Chem. Soc. 2007, 129, 33393347.

(18) Nelson, H. D.; et al. Mid-Gap States and Normal vs Inverted Bonding in Luminescent $\mathrm{Cu}+-$ and $\mathrm{Ag}+-$ Doped CdSe Nanocrystals. J. Am. Chem. Soc. 2017, 139, 6411-6421.

(19) Pinchetti, V.; et al. Excitonic Pathway to Photoinduced Magnetism in Colloidal Nanocrystals with Nonmagnetic Dopants. Nat. Nanotechnol. 2018, 13, 145-151.

(20) Mocatta, D.; et al. Heavily Doped Semiconductor Nanocrystal Quantum Dots. Science 2011, 332, 77-81.

(21) Sahu, A.; et al. Electronic Impurity Doping in CdSe Nanocrystals. Nano Lett. 2012, 12, 2587-2594.

(22) Amit, Y.; Li, Y.; Frenkel, A. I.; Banin, U. From Impurity Doping to Metallic Growth in Diffusion Doping: Properties and Structure of Silver-Doped InAs Nanocrystals. ACS Nano 2015, 9, 10790-10800.

(23) Kroupa, D. M.; et al. Synthesis and Spectroscopy of SilverDoped PbSe Quantum Dots. J. Am. Chem. Soc. 2017, 139, 1038210394.

(24) Shen, Q.; Liu, Y.; Xu, J.; Meng, C.; Liu, X. Microwave Induced Center-Doping of Silver Ions in Aqueous CdS Nanocrystals with Tunable, Impurity and Visible Emission. Chem. Commun. 2010, 46, $5701-5703$

(25) Son, D. H.; Hughes, S. M.; Yin, Y.; Alivisatos, A. P. Cation Exchange Reactions in Ionic Nanocrystals. Science 2004, 306, 10091012.

(26) Liu, J.; Zhao, Q.; Liu, J.-L.; Wu, Y.-S.; Cheng, Y.; Ji, M.-W.; Qian, H.-M.; Hao, W.-C.; Zhang, L.-J.; Wei, X.-J.; Wang, S.-G.; Zhang, J.-T.; Du, Y.; Dou, S.-X.; Zhu, H.-S. Heterovalent-Doping-Enabled Efficient Dopant Luminescence and Controllable Electronic Impurity Via a New Strategy of Preparing II-VI Nanocrystals. Adv. Mater. 2015, 27, 2753-2761.

(27) Delikanli, S.; et al. Mn2+-Doped CdSe/CdS Core/Multishell Colloidal Quantum Wells Enabling Tunable Carrier-Dopant Exchange Interactions. ACS Nano 2015, 9, 12473-12479.

(28) Liu, Y.-H.; Wayman, V. L.; Gibbons, P. C.; Loomis, R. A.; Buhro, W. E. Origin of High Photoluminescence Efficiencies in CdSe Quantum Belts. Nano Lett. 2010, 10, 352-357.

(29) Tessier, M. D.; et al. Spectroscopy of Colloidal Semiconductor Core/Shell Nanoplatelets with High Quantum Yield. Nano Lett. 2013, 13, 3321-3328.

(30) Polovitsyn, A.; et al. Synthesis of Air-Stable CdSe/ZnS CoreShell Nanoplatelets with Tunable Emission Wavelength. Chem. Mater. 2017, 29, 5671-5680.

(31) Meinardi, F.; et al. Highly Efficient Large-Area Colourless Luminescent Solar Concentrators Using Heavy-Metal-Free Colloidal Quantum Dots. Nat. Nanotechnol. 2015, 10, 878-885.

(32) Tessier, M. D.; Javaux, C.; Maksimovic, I.; Loriette, V.; Dubertret, B. Spectroscopy of Single CdSe Nanoplatelets. ACS Nano 2012, 6, 6751-6758.

(33) Khan, A. H.; et al. Near-Infrared Emitting Colloidal PbS Nanoplatelets: Lateral Size Control and Optical Spectroscopy. Chem. Mater. 2017, 29, 2883-2889.

(34) Achtstein, A. W.; et al. Linear Absorption in CdSe Nanoplates: Thickness and Lateral Size Dependency of the Intrinsic Absorption. J. Phys. Chem. C 2015, 119, 20156-20161.

(35) Gao, Y.; Weidman, M. C.; Tisdale, W. A. CdSe Nanoplatelet Films with Controlled Orientation of their Transition Dipole Moment. Nano Lett. 2017, 17, 3837-3843. 
(36) Scott, R.; et al. Directed Emission of CdSe Nanoplatelets Originating From Strongly Anisotropic 2D Electronic Structure. Nat. Nanotechnol. 2017, 12, 1155-1160.

(37) Ma, X.; et al. Anisotropic Photoluminescence from Isotropic Optical Transition Dipoles in Semiconductor Nanoplatelets. Nano Lett. 2018, 18, 4647-4652.

(38) Bertrand, G. H. V.; Polovitsyn, A.; Christodoulou, S.; Hossain Khan, A.; Moreels, I. Shape Control of Zincblende CdSe Nanoplatelets. Chem. Commun. 2016, 52, 11975-11978.

(39) Christodoulou, S.; et al. Chloride-Induced Thickness Control in CdSe Nanoplatelets. Nano Lett. 2018, 18, 6248-6254.

(40) Morgan, D.; Kelley, D. F. Role of Surface States in SilverDoped CdSe and CdSe/CdS Quantum Dots. J. Phys. Chem. C 2018, 122, 10627-10636.

(41) Ott, F. D.; Spiegel, L. L.; Norris, D. J.; Erwin, S. C. Microscopic Theory of Cation Exchange in CdSe Nanocrystals. Phys. Rev. Lett. 2014, 113, 156803.

(42) Yarema, M.; et al. Infrared Emitting and Photoconducting Colloidal Silver Chalcogenide Nanocrystal Quantum Dots from a Silylamide-Promoted Synthesis. ACS Nano 2011, 5, 3758-3765.

(43) Kambhampati, P. Unraveling the Structure and Dynamics of Excitons in Semiconductor Quantum Dots. Acc. Chem. Res. 2011, 44, $1-13$.

(44) Califano, M.; Gómez-Campos, F. M. Universal Trapping Mechanism in Semiconductor Nanocrystals. Nano Lett. 2013, 13, 2047-2052.

(45) Lorenzon, M.; et al. Reversed Oxygen Sensing Using Colloidal Quantum Wells Towards Highly Emissive Photoresponsive Varnishes. Nat. Commun. 2015, 6, 6434.

(46) Brovelli, S.; Galland, C.; Viswanatha, R.; Klimov, V. I. Tuning Radiative Recombination in $\mathrm{Cu}$-Doped Nanocrystals via Electrochemical Control of Surface Trapping. Nano Lett. 2012, 12, 43724379.

(47) She, C.; et al. Red, Yellow, Green, and Blue Amplified Spontaneous Emission and Lasing Using Colloidal CdSe Nanoplatelets. ACS Nano 2015, 9, 9475-9485.

(48) Li, Z.; et al. Uniform Thickness and Colloidal-Stable CdS Quantum Disks with Tunable Thickness: Synthesis and Properties. Nano Res. 2012, 5, 337-351.

(49) Ithurria, S.; Talapin, D. V. Colloidal Atomic Layer Deposition (c-ALD) using Self-Limiting Reactions at Nanocrystal Surface Coupled to Phase Transfer between Polar and Nonpolar Media. J. Am. Chem. Soc. 2012, 134, 18585-18590. 